\title{
Reamed intramedullary exchange nailing in the operative treatment of aseptic tibial shaft nonunion
}

\author{
Christian Hierholzer $^{1}$ - Jan Friederichs ${ }^{2}$ - Claudio Glowalla ${ }^{3}$ - Alexander Woltmann ${ }^{2}$. \\ Volker Bühren $^{2}$ • Christian von Rüden ${ }^{2,4}$
}

Received: 22 March 2016 / Accepted: 12 October 2016 / Published online: 28 October 2016

(C) The Author(s) 2016. This article is published with open access at Springerlink.com

\begin{abstract}
Purpose The aim of this study was to evaluate a standardized treatment protocol regarding the rate of secondary bone union, complications, and functional outcome.

Methods This study was started as a prospective study in a single Level I Trauma Centre between 2003 and 2012. The study group consisted of 188 patients with the diagnosis of an aseptic tibial shaft nonunion. Exchange nailing was performed following a standardized surgical protocol. Long-term followup was analyzed for rate of bone healing and functional outcome.

Results Osseous healing was achieved in 182 out of 188 patients $(97 \%)$ ). In 165 out of 188 patients ( $88 \%$ ), bone healing was observed timely and uneventfully after a single exchange nailing procedure. An open approach was necessary in 32 patients $(17 \%)$. Twenty-three patients $(12 \%)$ required additional therapy such as extracorporeal shock wave therapy. Post-operative complications were observed in seven patients (4\%). Almost all patients demonstrated osseous healing within 12 months, with the majority of osseous healing occurring within six months. A relevant shortening of the fractured tibia
\end{abstract}

Christian von Rüden

christian.vonrueden@bgu-murnau.de

1 Department of Trauma Surgery, University Hospital Zurich, Zurich, Switzerland

2 Department of Trauma Surgery, Trauma Center Murnau, Professor Küntscher Str. 8, 82418 Murnau, Germany

3 Clinic of Orthopedics and Sports Orthopedics, Klinikum rechts der Isar, Technical University of Munich, Munich, Germany

4 Institute of Biomechanics, Paracelsus Medical University, Salzburg, Austria was observed in 20 out of 188 patients (11\%). After a median follow-up of 23 months (range 12-45 months), outcome was evaluated using the assessment system of Friedman/Wyman. In summary, 154 out of 188 patients ( $82 \%$ ) had a good functional long-term result.

Discussion Reamed intramedullary exchange nailing including correction of axis alignment is a safe and effective treatment of aseptic tibial shaft nonunion with a high rate of bone healing and a good radiological and functional long-term outcome.

Keywords Long-term outcome $\cdot$ Nonunion $\cdot$ Reamed intramedullary exchange nailing $\cdot$ Tibia

\section{Introduction}

The gold standard for the treatment of tibial shaft fractures is intramedullary nailing. The technique of closed reduction and intramedullary stabilization follows the principle of biological osteosynthesis and is considered a dynamic stabilization technique resulting in secondary bone healing. Despite progress in surgical techniques and modern implants, impaired bone healing remains a challenging problem, specifically at the tibia where there is limited soft tissue coverage and a high rate of open injuries. The incidence of tibial shaft nonunion or delayed union reported in the literature reaches up to $16.7 \%$ following intramedullary nailing of tibia shaft fractures [1-3].

Whereas it is impossible to control injury-dependent risk factors for the development of a tibial shaft nonunion such as an open fracture and severe soft tissue injury [4], treatmentrelated factors can be addressed. Several factors originating from poor nailing technique such as fracture gap, axis deviation, and the application of small diameter nails and interlocking bolts result in instability of the osteosynthesis 
[5]. Typically, these fractures are linked with impaired bone healing and present as hypertrophic nonunion as a consequence of insufficient and unstable fracture stabilization. However, the hypertrophic form of tibial shaft nonunion offers the possibility of closed nonunion treatment by applying the principle of exchange nailing to increase mechanical stability and biological stimulation of the bone. Several techniques of treatment have been reported for the treatment of tibial shaft nonunion, most of them with a small number of patients. It is generally agreed that exchange nailing represents the treatment of choice. For non-infected diaphyseal nonunion of the tibia, success rates of 76-96\% have been reported [6-9]. Several prognostic factors such as time between initial injury and exchange nailing, type of fixation, fracture configuration, and fracture type [10] have been identified. Fracture healing could be achieved after a single nail exchange operation in more than $80 \%$ of the nonunions $[7,11]$. However, healing rates of more than $90 \%$ have also been described for alternative methods such as expandable intramedullary implants or plating $[12,13]$.

In this clinical trial we evaluated our therapeutic concept of closed revision and reamed exchange nailing for the treatment of aseptic tibial shaft nonunion including removal of the intramedullary nail, limited reaming of the intramedullary canal, and insertion of an intramedullary nail that is larger in diameter than the removed nail, canal filling in size, and offers optimal stability for uneventful bone healing [14]. In cases with healed or stable fibula, in which the intact fibula causes mechanical blockage and prevents dynamization and compression of the tibial fracture, we performed fibula osteotomy as a standard procedure despite this being discussed controversially $[8,15,16]$.

The aim of this study was to evaluate this concept on a prospective and large series of patients and to determine if osseous healing of aseptic tibial shaft nonunion in a high percentage of nonunion can be achieved with the ability to perform correction of axis alignment.

\section{Patients and methods}

Between 2003 and 2012, a prospective cohort study was performed in a Level I Trauma Centre, and 188 patients were included who had been treated with intramedullary nailing of a tibial shaft fracture and had developed aseptic tibial shaft nonunion. Written informed consent was obtained from all individual participants included in this study.

Fractures of the proximal and distal fifth of the tibia were primarily excluded from this study. Nonunion was determined clinically and radiologically at least 6 months after the index operation. Clinical signs included persistent pain with weight bearing, and radiological nonunion formation was determined as a lack of radiographic bridging of at least three out of four cortices assessed on antero-posterior (AP) and lateral conventional radiologic views. In cases of doubt, a CT scan was performed to detect radiological nonunion with formation of callus at all four cortices according to Heckman's criteria of fracture healing [17]. Patients with previous or consecutive positive bacterial cultures were excluded from this study to restrict the study group to aseptic tibia shaft nonunion.

Of the 188 patients included in this study, $165(88 \%)$ were referred for treatment of the tibial shaft nonunion from an outside institution, $23(12 \%)$ were initially treated at our institution. The study group consisted of $156(83 \%)$ male and $32(17 \%)$ female patients with a median age of 43 years (range 16-82). Forty-three out of 188 (23\%) patients suffered tibial shaft fractures as part of a polytrauma injury. Mechanism of injury included motor bike injuries in 82 patients (44\%), car accidents in 54 patients ( $29 \%$ ), sports injuries in 28 patients (14\%), and falls from a height over $3 \mathrm{~m}$ in 24 patients $(13 \%)$. Nonunion was classified as hypertrophic in 164 patients $(87 \%)$ and atrophic/ oligotrophic in 24 patients (13\%). The time between index operation and revision surgery was $<$ six months in 56 patients ( $30 \%$ ), between six and 12 months in 87 patients $(46 \%)$, and $>12$ months in 45 patients ( $24 \%$ ). More clinical details are summarized in Table 1.

In our standard surgical procedure the patient was positioned in supine position on a radiolucent operating table. After removal of the nail, care was taken to precisely position the guide wire in the center of the distal tibia. For this purpose, it is helpful to aim at the mid-talar region under fluoroscopic control using AP and lateral views. If both the correct insertion point and central endpoint of the nail are selected correctly, good axis alignment of the tibia shaft can be expected after successful tibia nailing.

Table 1 Summary of clinical characteristics of 188 patients with aseptic nonunion of the tibial shaft

\begin{tabular}{ll}
\hline Age [years], median [range] & $43(16-82)$ \\
AO-classification: & \\
type A1 & $32(17 \%)$ \\
type A2 & $41(22 \%)$ \\
type A3 & $30(16 \%)$ \\
type B & $62(33 \%)$ \\
type C & $23(12 \%)$ \\
Closed fractures: & $111(59 \%)$ \\
Open fractures: & \\
$\mathrm{I}^{\circ}$ & $17(9 \%)$ \\
II $^{\circ}$ & $38(20 \%)$ \\
III & $22(12 \%)$ \\
Index operation: & \\
unreamed & $147(78 \%)$ \\
reamed & $37(20 \%)$ \\
unknown & $4(2 \%)$ \\
\hline
\end{tabular}




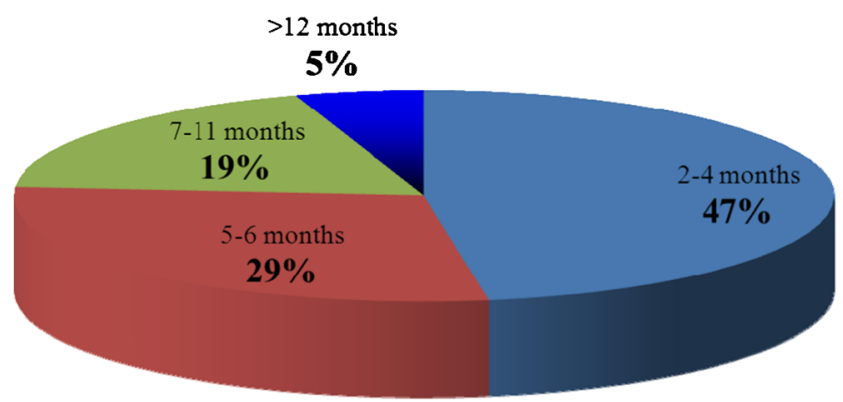

Fig. 1 Time to bone healing of 188 patients with a tibial shaft nonunion treated with reamed intramedullary exchange nailing. In only nine patients ( $5 \%$ ) time to osseous healing exceeded 12 months

Sequential reaming was performed using an incremental increase of drill bits with the goal of inserting an exchange nail with an increased diameter of at least $1 \mathrm{~mm}$ compared to the initial nail diameter size or at least $10 \mathrm{~mm}$ in diameter including interlocking screws with a diameter of $5 \mathrm{~mm}$. Since $5 \mathrm{~mm}$ interlocking screws significantly increased rotational stability compared to $4 \mathrm{~mm}$ interlocking screws [18], exchange nails with a diameter of $8 \mathrm{~mm}$ (with $4 \mathrm{~mm}$ interlocking screws) have not been used for revision surgery in this study. In our standard surgical procedure, all exchange nails were inserted using three interlocking screws distally, one interlocking screw and one compression screw proximally in the advanced locking mode. Even in cases with limited diameter of the intramedullary canal with primarily 7-8 mm unreamed nailing, exchange nails with a diameter of $10 \mathrm{~mm}$ were preferentially inserted.

The isthmal region in the intramedullary canal was overreamed with $1 \mathrm{~mm}$ more than the determined, final nail diameter. In the reaming process it was ensured that all exchange nails demonstrated good cortical contact, a snug fit and that any fracture gap or dehiscence was avoided. The reaming process was limited to a nail diameter that filled the intramedullary canal and was $2 \mathrm{~mm}$ larger than the previous nail. For exchange nailing, T2 ${ }^{\text {TM}}-$ Tibia nails (Stryker, Kalamazoo, Michigan, U.S.A.) were utilized offering the possibility of interfragmentary compression.
Therefore, distal interlocking screws were inserted according to both, manufacturer's instructions and to the findings that the greatest increases in torsional and bending stiffness of intramedullary nails were obtained by increasing the number of locking screws [19]. The tibial torsion was then assessed macroscopically and radiologically.

Consecutively, patients received physiotherapy and were mobilized out of bed. Following wound healing, weight bearing as tolerated was permitted. Until patients had resumed normal activity mobilization, subcutaneous antithrombotic medication was administered.

A radiologic follow-up study was performed after three to seven days following exchange nailing. Following discharge from hospital treatment, patients were followed up at regular office visits. Clinical assessment of wound healing, condition of soft tissues, and pain with weight bearing were recorded and sequential radiologic follow-up studies were requested at regular intervals at six and 12 weeks as well as six months post-operatively. Radiographs were assessed by a consultant of the Department of Radiology blinded to the patients' outcome. The median followup of patients was 23 months with a range from 12 to 45 months. Assessment of functional results was performed using the system of Friedman/Wyman including impairment in activities of daily life, range of motion of hip and knee joints, return to work, return to sports activities as prior to the injury, and pain assessment [20]. Results in this study are presented as median values.

\section{Results}

Exchange nailing was carried out as a closed procedure in 156 $(83 \%)$ of the patients whereas an open approach was necessary in $32(17 \%)$ of the patients. In 25 cases of an open approach (13\%), one unit of recombinant human Bone Morphogenetic Protein rhBMP-7 (OP- $1{ }^{\circledR}$, Stryker Biotech, Hopkinton, Massachusetts, U.S.A.) was applied in addition to bone grafting
Fig. 2 Tibial length discrepancy after exchange nailing of 188 patients with a tibial shaft nonunion treated with reamed intramedullary exchange nailing. An example of radiographic measurement of a tibial length discrepancy is demonstrated on the right side

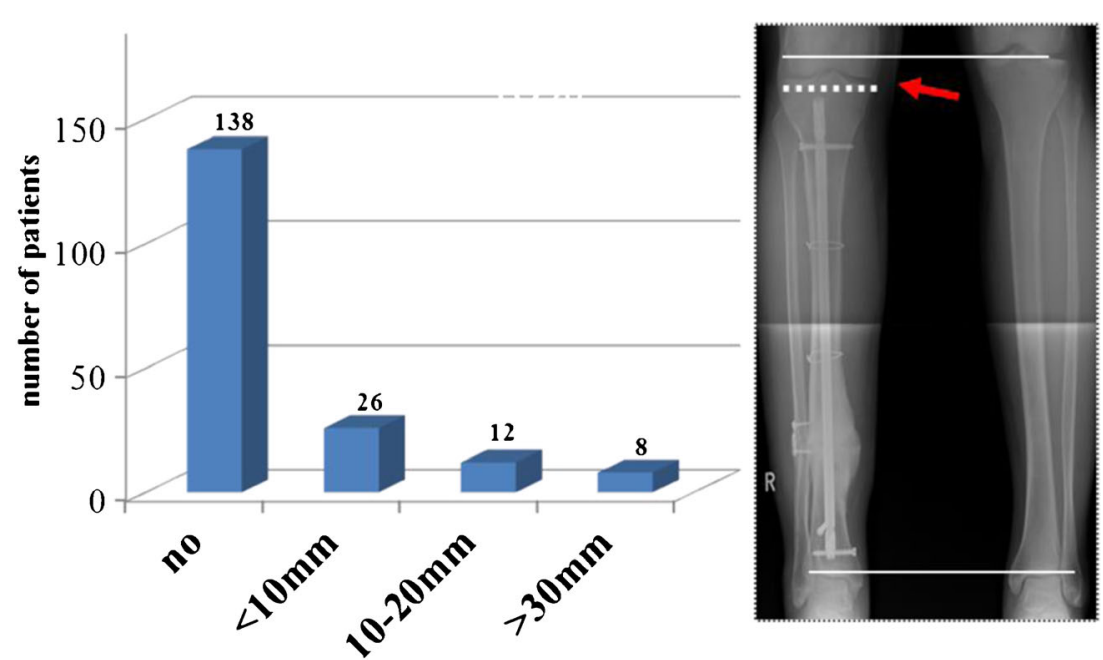


Fig. 3 Axial deviation after exchange nailing of tibial shaft nonunion treated with reamed intramedullary exchange nailing. Pre- and post-operative varus deviations are demonstrated in the left columns, valgus deviations in the right columns. An example of radiographic analysis is demonstrated on the right side

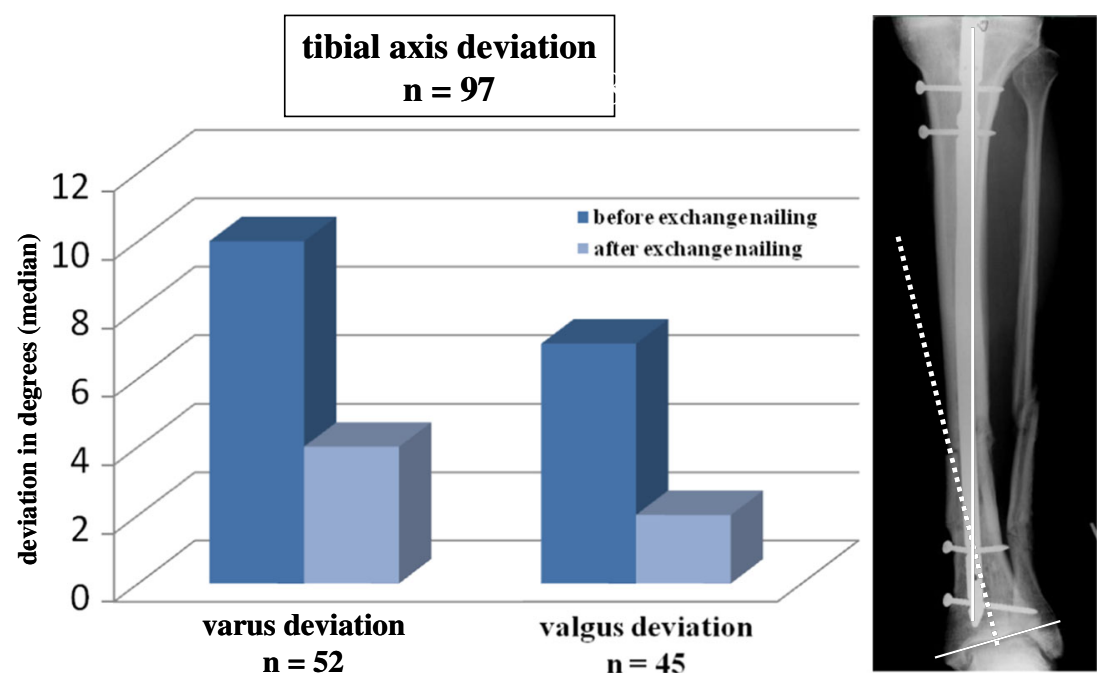

according to manufacturer's instructions and to Food and Drug Administration (FDA) approval. A fibular osteotomy was performed in 110 of the patients (59\%) when axial compression was applied and the manoeuvre was mechanically blocked by the fibular length. The median diameter of the extracted nail was $9 \mathrm{~mm}$ with a range of 8 to $11 \mathrm{~mm}$. In more than $90 \%$ of the inserted exchange nails, the diameter was $10 \mathrm{~mm}$ or more with a median of $11 \mathrm{~mm}$ and a range of $10 \mathrm{~mm}$ to $13 \mathrm{~mm}$. Dynamic compression was used in 162 of the patients $(86 \%)$. The advanced locking mode with axial compression on the dynamic interlocking bolt followed by insertion of an additional locking screw was applied in $21(11 \%)$ cases with a gap at the nonunion site in order to reduce the distance between the fracture ends. Static locking was observed in the remaining five patients.

Osseous healing was achieved in 182 out of 188 patients with aseptic tibial shaft delayed union and nonunion (97\%). In 165 patients ( $88 \%$ ), bone healing was achieved timely and uneventfully after a single exchange nailing procedure. Seventeen patients $(9 \%)$ required additional therapy, and in 11 cases one or more additional operations including multiple exchange nailing (three patients), additional open approach with autologous bone grafting (two patients), or a secondary dynamization of the inlaying exchange nail (nine patients) were necessary. Extracorporeal shock wave therapy (ESWT) was applied in 15 cases ( $8 \%$ ) after exchange nailing. Almost all patients obtained osseous healing within 12 months, with the majority of bone healing occurring after 6 months (Fig. 1). Postoperative complications were observed in eight patients (4\%). Surgical management was necessary in two cases of impaired wound healing with uneventful recovery and in two cases of haematoma at the iliac bone crest after autologous bone grafting, and in one case a compartment syndrome had to be treated surgically. A deep venous thrombosis was diagnosed in three patients although prophylaxis had correctly been applied.

After bone healing of the tibial nonunion, leg length was clinically assessed and bilateral tibial length was radiologically measured in all patients. A relevant shortening of the tibia was only observed in 20 out of 188 patients $(11 \%)$ as demonstrated in Fig. 2. Secondary osteotomy lengthening with distraction osteogenesis was performed in eight patients with tibial length discrepancy of more than $30 \mathrm{~mm}$ (Fig. 2). Coronal axis alignment was assessed by measuring the mechanical and anatomical axis of the tibia on digitalized conventional x-ray imaging studies using standard software. As demonstrated in Fig. 3, a varus axis deviation with a median of $10^{\circ}$ (range 2-35) was observed in 52 patients while a valgus axis deviation with a median of $7^{\circ}$ (range 2-20) was measured in 44 patients after primary intramedullary nailing of a tibial shaft fracture. A correction of axis was possible in almost all cases with a median deviation of only $2^{\circ}$ after revision surgery.

For assessment of torsional axis deviation, patients who demonstrated a clinically apparent discrepancy of torsion compared to the unaffected leg underwent a computedtomographic analysis of torsion. Pre-operatively, a total of 18 patients $(10 \%)$ with a relevant torsional deviation were identified. Thirteen of them had an external torsional axis deviation with a median of $15^{\circ}$ (range 3-28) while five patients demonstrated an internal torsional axis deviation with a

Table 2 Functional long-term results according to the assessment system of Friedman/Wyman [20] after a median follow-up of 23 (range 1245 ) months of 188 patients with aseptic tibial shaft nonunion

\begin{tabular}{llll}
\hline Functional assessment & good & fair & poor \\
& $154(82 \%)$ & $18(10 \%)$ & $16(8 \%)$ \\
Impairment of ADL & none & mild & moderate \\
& $128(68 \%)$ & $38(20 \%)$ & $22(12 \%)$ \\
Loss of hip or knee $\mathrm{ROM}^{\mathrm{b}}$ & $<20 \%$ & $20-50 \%$ & $>50 \%$ \\
& $150(79 \%)$ & $37(20 \%)$ & $1(1 \%)$ \\
Pain & none & mild/moderate & severe \\
& $118(63 \%)$ & $68(36 \%)$ & $2(1 \%)$ \\
\hline
\end{tabular}

${ }^{\mathrm{a}} A D L$ activities of daily living

${ }^{\mathrm{b}} R O M$ range of motion 
median of $15^{\circ}$ (range 2-25). If possible, a correction of the torsional axis deviation was performed; however, no routine computed-tomographic analysis was conducted and thus, no post-operative data was available.

In addition to bone healing and axis alignment after a median follow-up of 23 (range 12-45) months, functional longterm outcome was evaluated [20]. As demonstrated in Table 2, 154 out of 188 patients ( $82 \%$ ) had a good functional longterm result.

\section{Discussion}

In recent literature, nonunion was the most prevalent complication of tibial shaft fractures and had been developed in up to $27 \%$ of patients, independent of different fixation methods such as intramedullary nailing, locking compression plating, or external fixation [21-24]. Evidence comparing reamed with unreamed intramedullary nailing for closed tibial fractures indicates that reamed intramedullary nailing may lead to significantly lower risk for nonunion, screw failure, implant exchange, and dynamization without increasing operative complications [21, 25], whereas the choice for open tibial fractures remains uncertain [26].

In our study, a standardized protocol of reamed exchange nailing proved to be an effective and safe method in the treatment of aseptic tibial shaft nonunion. In $83 \%$ of cases, closed nonunion treatment was performed without surgical opening of the nonunion site. Reamed exchange nailing and dynamic compression of the nonunion site resulted in increased stability with the possibility of early and unrestricted weight bearing, high patient comfort with little pain and discomfort, as well as good functional outcome [21]. Key steps for successful osseous healing include closed nonunion treatment, correction of axis deviation, limited reaming and biological augmentation by internal reaming graft, increased rotational and axial stability by insertion of an increased nail diameter, and by dynamic compression of the nonunion site. Furthermore, the mechanical stability of intramedullary nailing can be affected by various locking parameters such as number of screws, distance and orientation between screws, blocking of screws, and the surgical technique of freehand locking [19].

In our study, the mechanical advantage of the increased nail diameter represented the key feature. Penzkofer et al. demonstrated that the axial and rotational stability significantly increased with an increase in nail diameter. In addition, the application of interlocking screws with a diameter of $5 \mathrm{~mm}$ significantly augmented rotational stability compared to $4 \mathrm{~mm}$ diameter interlocking screws [18]. Insertion of the interlocking screws using a free hand technique results in jamming of the interlocking screw with the nail and with cortical bone, providing inherent angular stability of the construct [27]. This concept is supported by a study on femoral nonunion where a positive correlation between reaming diameter and nail size of at least $2 \mathrm{~mm}$ larger than the primary nail and the fracture union rate was demonstrated by $\mathrm{Wu}$ [28]. Additionally, the distribution of axial forces and compression at the nonunion site is very important. This can be ensured by applying dynamic compression through the insertion of an interlocking and a compression screw. Post-operative unrestricted weight bearing also contributes to axial compression of the nonunion site.

However, a major concern of the reaming process is the detrimental effect of heat created by the drill bit resulting in necrosis and impairment of bone healing. In order to prevent excessive heat during exchange nailing, a sharp reamer was utilized and the reaming process was limited to a nail diameter that filled the intramedullary canal and was $2 \mathrm{~mm}$ larger than the previous nail. An advantageous effect of the first two to three initial reaming steps is the debridement of the intramedullary canal. Fibrotic tissue is removed and can be irrigated. Interestingly, additional beneficial effects of limited reaming have been described recently. Reaming graft has been demonstrated to result in improvement of local biology and stimulation of bone healing at the nonunion site [21]. In addition, utilization of reaming debris instead of stem cells or autologous bone marrow grafting does not require an additional surgical procedure to harvest biological material [29]. Reaming debris contains viable osteoblast-like cells and growth factors, and therefore may act as a natural osteoinductive scaffold. In a sheep tibia model, bones treated with reaming debris showed larger callus volume, increased bone volume, and decreased cartilage volume in the fracture gap, as well as increased torsional stiffness compared to the unreamed group [30]. Other animal models suggested that unreamed and limited reamed intramedullary nails provide improved healing of tibia fractures compared to extensively reamed nails [31]. Limited reaming may induce angiogenesis and may therefore be beneficial for stimulating the amount of bone formation around a critically-sized defect. Several studies have demonstrated that the direction of blood flow reversed from centrifugal to centripetal after loss of the endosteal supply results in a sixfold increase in the periosteal flow compared to the control group composed of unreamed contralateral tibiae [32]. In addition, limited reaming may improve blood flow in the surrounding soft tissues as demonstrated by Schemitsch et al., who conducted a fractured sheep tibia model and found that perfusion in the surrounding tibia muscle was significantly greater in the reamed group compared to the unreamed group [33]. In order to prevent excessive heat during exchange nailing, a sharp reamer should be utilized and the reaming process should be limited to a nail diameter that fills the intramedullary canal and is $2 \mathrm{~mm}$ larger than the previous nail.

An additional unique property of the lower leg is the double bone structure with both tibial and fibular bones. Often, rapid osseous healing of the fibula precedes the tibial bone healing 
causing a mechanical blockage which prevents dynamization and compression of the tibial fracture. The question if and when a fibula osteotomy should be performed is controversially discussed $[8,15,16]$. In cases of tibia nonunion with interfragmentary dehiscence or gap formation, a healed fibula caused mechanical blockage and prevented dynamization and compression of the nonunion site. Therefore, in order to remove nonunion gap and apply interfragmentary compression at the nonunion site, a fibula osteotomy was performed. For fibula osteotomy we typically resect a fibula bone fragment of approximately $1 \mathrm{~cm}$ and apply dynamic tension band fixation with the intention of dynamically stabilizing the fibula fragments and providing axis alignment. Alternatively, oblique osteotomy of the fibula can be performed without bone resection. However, fibula healing may occur rapidly and fibula fusion may precede nonunion healing of the tibia resulting in recurrence of mechanical blocking and preventing nonunion compression.

Additional surgical therapy including multiple exchange nailing, additional open approach with autologous bone grafting, secondary dynamization and compression of the inlaying exchange nail, or additional conservative treatment such as ESWT [34] may be necessary in cases, in which nonunion healing was clinically and radiologically prolonged.

In conclusion, reamed intramedullary exchange nailing including correction of axis alignment as described in this study is a safe and effective treatment of aseptic tibial shaft nonunion with a high rate of bone healing and a good radiological and functional long-term outcome.

Acknowledgments Open access funding provided by Paracelsus Medical University, Austria.

Compliance with ethical standards All procedures performed in this study involving human participants were in accordance with the ethical standards of the institutional and national research committee and with the 1964 Helsinki declaration and its later amendments.

Conflict of interest The authors declare that they have no conflict of interest.

Open Access This article is distributed under the terms of the Creative Commons Attribution 4.0 International License (http:// creativecommons.org/licenses/by/4.0/), which permits unrestricted use, distribution, and reproduction in any medium, provided you give appropriate credit to the original author(s) and the source, provide a link to the Creative Commons license, and indicate if changes were made.

\section{References}

1. Attal R, Blauth $M$ (2010) Unreamed intramedullary nailing. Orthopade 39:182-191. doi:10.1007/s00132-009-1524-5

2. Forster MC, Bruce ASW, Aster AS (2005) Should the tibia be reamed when nailing? Injury 36:439-444

3. Lin J, Hou SM (2001) Unreamed locked tight-fitting nailing for acute tibial fractures. J Orthop Trauma 15(1):40-46
4. McQueen MM, Christie J, Court-Brown CM (1996) Acute compartment syndrome in tibial diaphyseal fractures. J Bone Joint Surg (Br) 78:95-98

5. Rodriguez-Merchan EC, Forriol F (2004) General principles and experimental data. Clin Orthop Relat Res 419:4-12

6. Swanson EA, Garrard EC, O Connor DP, Brinker MR (2015) Results of a systematic approach to exchange nailing for the treatment of aseptic tibial nonunion. J Orthop Trauma 29(1):28-35. doi:10.1097/BOT.0000000000000151

7. Court-Brown CM, Keating JF, Christie J, McQueen MM (1995) Exchange intramedullary nailing: its use in aseptic tibial nonunion. J Bone Joint Surg (Br) 77:407-411

8. Wu CC, Shih CH, Chen WJ, Tai CL (1999) High success rate with exchange nailing to treat a tibial shaft aseptic nonunion. J Orthop Tauma 13:33-38

9. Zelle BA, Gruen GS, Klatt B, Haemmerle MJ, Rosenblum WJ, Prayson MJ (2004) Exchange reamed nailing for aseptic nonunion of the tibia. J Trauma 57:1053-1059

10. Mercado EM, Lim EV, Stern P, Aquino NJ (2001) Exchange nailing for failure of initially rodded tibial shaft fractures. Orthopedics 24:757-762

11. Templeman D, Thomas M, Varecka T, Kyle R (1995) Exchange reamed intramedullary nailing for delayed union and nonunion of the tibia. Clin Orthop Relat Res 315:169-175

12. Niu Y, Bai Y, Xu S, Liu X, Wang P, Wu D, Zhang C, Li M (2011) Treatment of lower extremity long bone nonunion with expandable intramedullary nailing and autologous bone grafting. Arch Orthop Trauma Surg 131:885-891. doi:10.1007/s00402-010-1226-9

13. Wiss DA, Johnson DL, Miao M (1992) Compression plating for nonunion after failed external fixation of open tibial fractures. J Bone Joint Surg Am 74:1279-1285

14. Mayo KA, Benirschke SK (1990) Treatment of tibial malunions and nonunion with reamed intramedullary nails. Orthop Clin North Am 21(4):715-724

15. Bonnevialle P, Bellumore Y, Foucras L, Hézard L, Mansat M (2000) Tibial fracture with intact fibula treated by reamed nailing. Rev Chir Orthop Reparatrice Appar Mot 86:29-37

16. Hsiao CW, Wu CC, Su CY, Fan KF, Tseng IC, Lee PC (2006) Exchange nailing for aseptic tibial shaft nonunion: emphasis on the influence of a concomitant fibulotomy. Chang Gung Med J 29:283-290

17. Heckman JD, Ryaby JP, McCabe J, Frey JJ, Kilcoyne RF (1994) Acceleration of tibial fracture-healing by non-invasive, lowintensity pulsed ultrasound. J Bone Joint Surg Am 76:26-34

18. Penzkofer R, Maier M, Nolte A, von Oldenburg G, Püschel K, Bühren V, Augat P (2009) Influence of intramedullary nail diameter and locking mode on the stability of tibial shaft fracture fixation. Arch Orthop Trauma Surg 129:525-531

19. Hoffmann S, Gerber C, von Oldenburg G, Kessler M, Stephan D, Augat P (2015) Effect of angular stability and other locking parameters on the mechanical performance of intramedullary nails. Biomed Tech (Berl) 60(2):157-164. doi:10.1515/bmt-2014-0100

20. Friedman RJ, Wyman ET (1986) Ipsilateral hip and femoral shaft fractures. Clin Orthop Relat Res 208:188-194

21. Högel F, Gerber C, Bühren V, Augat P (2013) Reamed intramedullary nailing of diaphyseal tibial fractures: comparison of compression and non-compression nailing. Eur J Trauma Emerg Surg 39:73-77. doi:10.1007/s00068-012-0237-3

22. Piątkowski K, Piekarczyk P, Kwiatkowski K, Przybycień M, Chwedczuk B (2015) Comparison of different locking plate fixation methods in distal tibia fractures. Int Orthop 39(11):2245-2251. doi: 10.1007/s00264-015-2906-4

23. Fadel M, Ahmed MA, Al-Dars AM, Maabed MA, Shawki H (2015) Ilizarov external fixation versus plate osteosynthesis in the management of extra-articular fractures of the distal tibia. Int Orthop 39(3):513-519. doi:10.1007/s00264-014-2607-4

24. Sathiyakumar V, Thakore RV, Ihejirika RC, Obremskey WT, Sethi MK (2014) Distal tibia fractures and medial plating: factors 
influencing re-operation. Int Orthop 38(7):1483-1488. doi:10.1007 /s00264-014-2345-7

25. Xia L, Zhou J, Zhang Y, Mei G, Jin D (2014) A meta-analysis of reamed versus unreamed intramedullary nailing for the treatment of closed tibial fractures. Orthopedics 37(4):e332-e338. doi:10.3928 /01477447-20140401-52

26. Xue D, Zheng Q, Li H, Qian S, Zhang B, Pan Z (2010) Reamed and unreamed intramedullary nailing for the treatment of open and closed tibial fractures: a subgroup analysis of randomised trials. Int Orthop 34(8):1307-1313. doi:10.1007 /s00264-009-0895-x

27. Augat $P$, Bühren V (2015) Intramedullary nailing of the distal tibia. Does angular stable locking make a difference? Unfallchirurg 118(4):311-317

28. Wu CC (2007) Exchange nailing for aseptic nonunion of femoral shaft: a retrospective cohort study for effect of reaming size. J Trauma 63:859-865

29. Guimarães JA, Duarte ME, Fernandes MB, Vianna VF, Rocha TH, Bonfim DC, Casado PL, do Val Guimarães IC, Velarde LG, Dutra HS, Giannoudis PV (2014) The effect of autologous concentrated bone-marrow grafting on the healing of femoral shaft non-unions after locked intramedullary nailing. Injury 45 Suppl 5:S7-S13

30. Frölke JP, Bakker FC, Patka P, Haarman HJ (2001) Reaming debris in osteotomized sheep tibiae. J Trauma 50:65-69

31. Kuzyk PR, Li R, Zdero R, Davies JE, Schemitsch EH (2011) The effect of intramedullary reaming on a diaphyseal bone defect of the tibia. J Trauma 70:1248-1256. doi:10.1097/TA.0b013e3181e985bd

32. Reichert IL, McCarthy ID, Hughes SP (1995) The acute vascular response to intramedullary reaming. Microsphere estimation of blood flow in the intact ovine tibia. J Bone Joint Surg (Br) 77: 490-493

33. Schemitsch EH, Kowalski MJ, Swiontkowski MF (1996) Softtissue blood flow following reamed versus undreamed locked intramedullary nailing: a fractured sheep tibia model. Ann Plast Surg 36:70-75

34. Haffner N, Antonic V, Smolen D, Slezak P, Schaden W, Mittermayr R, Stojadinovic A (2016) Extracorporeal shockwave therapy (ESWT) ameliorates healing of tibial fracture non-union unresponsive to conventional therapy. Injury 47(7):1506-1513. doi:10.1016 /j.injury.2016.04.010 